\title{
A quantitative measure for the uniformity of fixation density: The Voronoi method
}

\author{
EELCO A. B. OVER, IGNACE T. C. HOOGE, and CASPER J. ERKELENS \\ Utrecht University, Utrecht, The Netherlands
}

\begin{abstract}
In order to characterize the uniformity of fixation density, we propose a quantitative measure based on Voronoi diagrams, in which cells are defined around fixation locations. We examined how normalized cell size distributions are related to homogeneous and inhomogeneous fixation densities. Two possible measures for use with the Voronoi method are discussed. Both show good correlation with subjective visual evaluations of the uniformity of fixation densities. Not only are these measures objective and quantitative, they also have a simple intuitive meaning: They may be thought of as reflecting the clustering of fixations.
\end{abstract}

Scanning the visual environment is an ongoing activity of human beings. Visual scanning consists of saccadic eye movements interleaved with periods of fixation. The spatial distribution of fixation locations and its relation to scene content and task are interesting because they may illustrate how the viewer interprets the scene. During various search tasks, for example, people were found to fixate objects rather than the space between objects (Hooge \& Erkelens, 1999; Williams, 1966). However, Zelinsky, Rao, Hayhoe, and Ballard (1997) found fixations between objects as well and challenged this view. Since the pioneering work by Yarbus (1967), sequences of visual fixation, later called scan paths (Noton \& Stark, 1971a, 1971b), have been generally accepted representations of the viewing behavior of observers. Using scan paths, researchers have demonstrated that schizophrenics (Phillips \& David, 1997) and people with brain damage (Zihl \& Hebel, 1997) exhibit viewing behavior different from that of normal, healthy people. De Lucio, Mohamadian, Ruiz, Banayas, and Bernaldez (1996) have shown differences in scanning behavior between men and women. Zangemeister, Sherman, and Stark (1995) have described differences both between processes of viewing realistic and abstract art and between the viewing behavior of professional and nonprofessional art viewers. All of these reports referred to temporal differences in viewing behavior (e.g., in fixation duration or order of fixation). Reports referring to spatial differences in viewing behavior, on the other hand, are scarce. This gap in the literature may be caused by the absence of quantitative and objective methods that can be used for characterizing spatial aspects of viewing be-

We thank Keith Rayner and an anonymous reviewer for their helpful comments on an earlier version of the manuscript. E.A.B.O. was supported by TNO Human Factors, Soesterberg, The Netherlands. Correspondence relating to this article may be sent to E. A. B. Over, Physics of Man, Helmholtz Institute, Utrecht University, Princetonplein 5, 3584 CC Utrecht, The Netherlands (e-mail: e.over@erasmusmc.nl). havior. Here we introduce the Voronoi method, which is based on Voronoi diagrams (Voronoi, 1908). The Voronoi method is a quantitative and objective method for characterizing fixation density, and we show that it may be a useful tool in the analysis of spatial viewing behavior.

Scan paths capture most features of viewing behavior, because they provide information about both the temporal and the spatial domains. However, analysis of scan paths is usually restricted to subjective, visual inspection. As an exception, Choi, Mosley, and Stark (1995) developed a quantitative method to compare individual scan paths: the string analysis. This method was also used to compare scan paths made during first-time visual inspection with eye movement trajectories made later during imagery of the same scene (Brandt \& Stark, 1997; Gbadamosi \& Zangemeister, 2001). The string analysis converts scan paths to strings of characters: Each character represents a particular part of the stimulus that has been fixated. After removal of all refixations of the same area, strings are compared to a reference string, and a distance from the scan path to the reference string is calculated. Finally, normalization of this distance by the length of the string yields a number between 0 (identical scan paths) and 1 (completely different scan paths). This analysis is suitable for comparing individual scan paths, and it can also be used to compare scan paths that are generated by computer models to a human reference scan path. However, a disadvantage of the string analysis is that regions of the stimulus have to be designated to represent characters. Each of these regions is often called a region of interest (ROI) or area of interest (AOI). The subdivision into ROIs (see, e.g., Gbadamosi \& Zangemeister, 2001; Laeng \& Teodorescu, 2002; Pelz, Hayhoe, \& Loeber, 2001; Shinoda, Hayhoe, \& Shrivastava, 2001) allows for comparisons of the same feature, for example dwell time. The designation of suitable ROIs usually will not be a problem if the stimulus results in a clustering of fixations, such as with stimuli that consist of a collection of items (Zelinsky et al., 1997). 
In Brandt and Stark's (1997) study, even the stimuli themselves could be used to define ROIs because of the spatial arrangement of subfeatures. Designating different ROIs is more difficult, however, when clustering of fixations does not occur (e.g., when there are no clear separate regions in the stimulus, like a white car in the mist). If it is difficult to designate ROIs, it may even be difficult to determine the number of ROIs. If this is the case, it may be more useful to characterize global densities of fixations in order to investigate aspects of their spatial distribution.

Comparing individual scan paths is one thing the researcher can do. Comparing viewing behavior, however, requires comparing sets of scan paths (possibly of different sizes) to one another. One of the major problems in this kind of eye movement research is to distill useful information out of the huge amount of available data. Some important requirements for an objective and quantitative analysis are the independence of (1) stimulus size, (2) number of trials, (3) number of fixations in each trial, and (4) (possibly) number of subjects. In this article, we concentrate on a single property of viewing behavior that meets the requirements mentioned above: the density of fixation locations. This single feature allows for easy comparisons of large data sets (e.g., sets of patients and controls) and is informative about the spatial aspects of viewing behavior.

A mathematically elegant procedure to characterize densities is to construct Voronoi diagrams (Voronoi, 1908). The Voronoi diagram is a division of an area into cells that are associated with particular points within that area. A cell is defined by the area taken up by the points that are closer to one particular point than to any other. In our case, the particular points are the fixation locations. The Voronoi diagram constructed for fixation locations has small cells in parts of the stimulus that are fixated frequently and large cells in parts of the stimulus that are fixated occasionally. Distributions of cell sizes therefore correlate to distributions of fixation densities.

In the first section of this article, we will explain how Voronoi diagrams can be used to characterize the uniformity of fixation density. In the second section, we apply the Voronoi diagram method to eye movement data in order to evaluate the outcome. In the third section, we describe simulations performed to get a feeling for the outcomes and requirements of the Voronoi method, and we end by describing our conclusions.

\section{THE VORONOI METHOD}

Areas can be filled with patches in numerous ways. The patches can be regular, as in a mosaic, for example. Or they can be irregular, as in the pattern that emerges when mud is dried and cracked by the sun. The Voronoi tessellation is an example of such an irregular filling of an area.

\section{Construction of Voronoi Diagrams}

Figure 1 systematically shows in a number of steps the construction of a Voronoi diagram for a set of fixation locations. It depicts the procedure of creating the Voronoi cell that belongs to the first fixation location (panel A): To begin, connecting lines are drawn to all other fixation locations (panel B). For reasons of clarity, we have only drawn the connecting lines that are relevant for this particular cell. The next step is that we compute the perpendicular lines (dashed) that bisect the connecting lines (panel C). The smallest area enclosed by these perpendicular lines is the Voronoi cell for the first fixation location (panels D and $\mathrm{E}$ ). The procedure is repeated for all fixation locations (panel F). Voronoi cells for fixation locations neighboring the stimulus border are partly bounded by the edge of the stimulus. The sum of all cell areas equals the total stimulus area.

Our method focuses on one of the properties of Voronoi diagrams - namely, the size of cells. The size of Voronoi cells depends on the size of the stimulus, the number of fixations per trial, and the location of fixations relative to other fixations. Figure 2 shows three hypothetical trials with symmetrical patterns of fixations that differ in these parameters. In this example, we present symmetrical patterns because they are simple and regular, but the same reasoning also applies to irregular patterns. Intuitively, in all three examples of Figure 2, the fixations are evenly scattered over the stimulus area. This is also clear from the fact that the Voronoi cells have equal size in each example. In order to match this intuition, the outcomes of the Voronoi method should be equal for all three patterns of fixation locations in Figure 2. Neither the size of the stimulus nor the number of fixations should affect the outcome of the method; to achieve this, the size of the cells should be normalized to correct for these two factors. Dividing the individual cell sizes by the mean cell size accomplishes this correction for both the number of fixations and the size of the stimulus, yielding a distribution of normalized cell sizes (dimensionless) with a mean of exactly 1 . Because the distribution of normalized cell sizes of every trial has an average value of 1 , the summation of all normalized cell size distributions also has an average value of 1 . A wide distribution represents very different cell sizes, and therefore an irregular pattern of fixations. A narrow distribution represents cells of approximately the same size, and therefore a homogeneous distribution of fixations.

\section{Discussion}

It may seem like a lot of work to construct Voronoi diagrams for each separate trial, but the Voronoi routine is included in many mathematical software packages (for example, Mathematica and MATLAB). Mathematica includes the option to build bounded Voronoi diagrams like the ones described above (where cells are limited by the stimulus boundaries). If only unbounded Voronoi diagrams are available (e.g., in MATLAB), the cells for all fixation locations can be calculated using the following simple trick.

1. Mirror all fixation locations relative to all stimulus boundaries (which must be straight lines).

2. Construct the unbounded Voronoi diagram for the collection of real and mirrored fixation locations. 
A

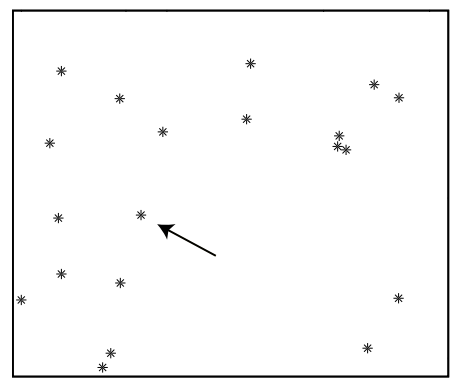

D

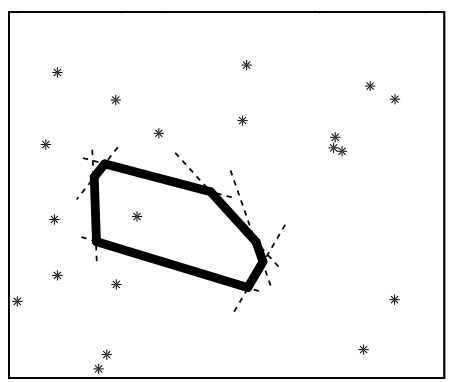

B

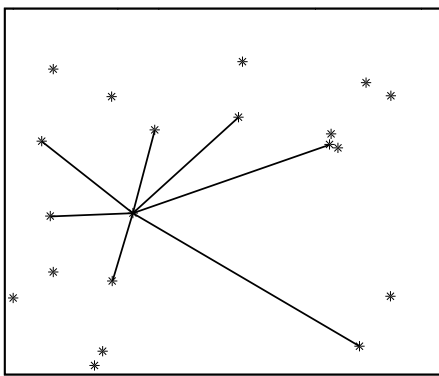

$\mathrm{E}$

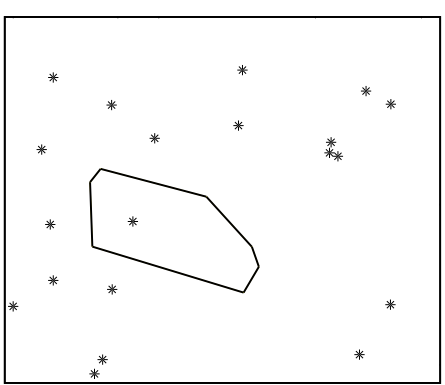

C

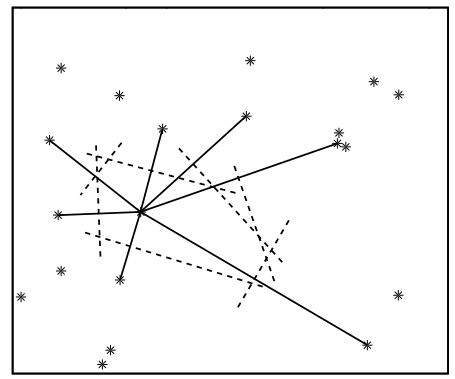

$\mathrm{F}$

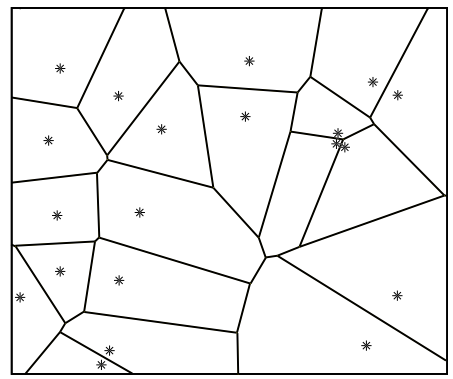

Figure 1. Construction of the Voronoi diagram. Pick a single fixation location (A) and draw connecting lines to its neighboring fixation locations (B). Construct a perpendicular line bisecting each connecting line (C). The smallest area enclosed by these perpendicular lines (D) is the Voronoi cell (E). Repeating this procedure for each fixation location yields the Voronoi diagram (F).

3. Remove the cells belonging to the mirrored fixation locations from the analysis.

For the Voronoi method to work properly, there are some requirements for the data. These requirements are not strict, though, and usually they are already met.

1. There must be fixation locations. Since Voronoi diagrams are calculated from discrete points, a scan path that entirely consists of smooth pursuit would be unsuitable for this method.

2. Fixation locations must be unique; that is, a fixation should not be at the exact same location as any other fixation. In most eye movement data, two fixation locations will not fall on the exact same location. This is merely a question of the accuracy of the eye movement recording equipment and analysis method: A higher spatial resolution during recording and more decimal places in the analysis reduce the probability of identical fixation locations. Moreover, fixation locations are usually the result of averaging a number of samples, which further decreases the possibility of identical fixation locations.

3. Fixation locations must be within the boundaries of an area that has to be chosen prior to the analysis. It is logical to choose the field of view relevant to the current task, or otherwise, the smallest region that contains all eye movements is a good choice for setting boundaries. This region should be the same for all trials of the experiment.

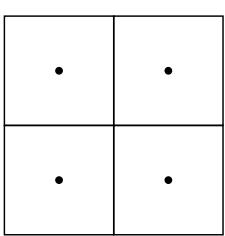

A

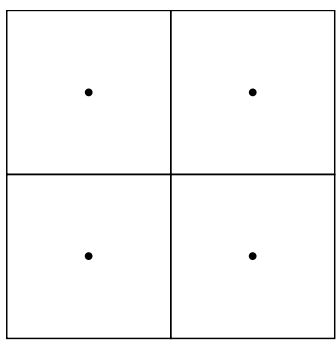

B

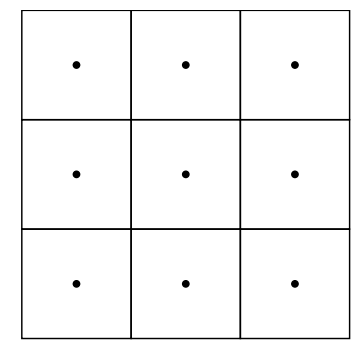

C

Figure 2. Three simple patterns of fixations and their Voronoi diagrams. Using panel $B$ as a reference, panel $A$ has the same number of fixations but a different stimulus size, and panel $\mathrm{C}$ has the same stimulus size but a different number of fixations. The three fixation densities, though, are all equally uniform. 
Our method depends on only one arbitrary choicenamely, the size (and shape) of the relevant part of the field of view. In most laboratory experiments, these are of course identical to the stimulus boundaries; in daily life, they should be defined by the current task. Imposing this restriction is not an unreasonable thing to do, because in most experiments, and even in daily life, eye movements are restricted to some part of the total field of view (e.g., a computer monitor). The choice of a field of view does not lead to a loss of information, because it can still be used as an indicator for the scale of the total scan path, and this information is retained in the normalization procedure: A multiplication of the average Voronoi cell size and the number of fixations gives the total stimulus size.

The Voronoi method is a purely spatial method. All temporal information included in a scan path is discarded when the scan path is converted to a collection of fixation locations. However, temporal information could possibly be included in the Voronoi method as the third dimension, in addition to the two spatial dimensions. Consequently, the cells of such a three-dimensional Voronoi diagram would themselves be three-dimensional, raising the questions: How should a spatial dimension be scaled to a temporal dimension, and what would the volume of such a three-dimensional cell represent? These issues would have to be investigated thoroughly before the method could be extended to three dimensions in order to include the temporal aspect of scan paths.

We will now apply the Voronoi method to an eye movement experiment with three conditions that we expected to yield very different uniformities of fixation density.

\section{EYE MOVEMENT EXPERIMENT}

To test our Voronoi method, we designed an experiment with three conditions in which we expected to find uniformities of fixation density that were very different from each other. Afterward, this expectation was confirmed by subjective visual evaluation of local fixation densities. We used two tasks - free viewing and visual search. The stimuli were either highly homogeneous or highly structured images.

The first condition (homogeneous search) was designed so that fixation locations were likely to be spread evenly over the stimulus area. The task was to search for an unknown number of targets. The subjects were expected to search the whole stimulus area, because they did not know in advance the total number of targets that were present in the trial. The stimulus was a homogeneous gray field with targets that were slightly darker. In every part of a homogeneously colored search area it is equally difficult to find targets, so the stimulus itself does not favor any part of the stimulus being fixated more often than other parts. The task in the second condition (structured search) also was to find an unknown number of targets, but the stimulus was a grayscale portrait of a group of people. The search task again required the subject to inspect every part of the stimulus, but the highly structured background made it more difficult to find targets in some parts of the stimulus than in others, for instance because of crowding (Levi, Klein, \& Aitsebaomo, 1985; Westheimer \& Hauske, 1975). We therefore expected more fixations to occur in the difficult parts of the stimulus, for example parts with large contrast differences. In the third condition (free viewing), observers were not asked to search for targets, but merely to look at pictures. In this condition, there was no need to examine all parts of the stimulus, because observers were free to view the picture as they liked. We expected the structured nature of the stimulus to yield different fixation probabilities for different parts of the stimulus. These different probabilities could be due to differences in the stimuli themselves, such as differences in contrast. The interpretation of the stimulus might also affect its fixation probabilities, though: Interesting parts are more likely to be fixated than parts that are not.

The spatial distribution of fixations depends not only on the instruction and the stimulus properties, but also on idiosyncratic behavior of the subjects. For example, the search strategy (Aks, Zelinsky, \& Sprott, 2002; Zangemeister et al., 1995) and physical condition (De Gennaro, Ferrara, Curcio, \& Bertini, 2001) of the subject play important roles in the properties of eye movements, and consequently in the appearance of a scan path. We therefore expected to find some differences between subjects, but the average distributions of fixations would have to be as follows: (1) In the homogeneous search condition, we expected the fixations to be spread more or less homogeneously in the stimulus area, and therefore we expected cells of approximately the same size. (2) In the structured search condition, we expected the fixations to cluster more in some parts of the stimulus, and we expected cell sizes to be somewhat different. (3) In the free viewing condition, we expected the fixations to be strongly clustered in interesting parts of the stimulus, so cell sizes would be strongly different from each other in each trial.

We applied the Voronoi method to the three conditions to investigate whether the results would be in accordance with the predicted uniformity of fixation density. We expected the skewness of the histograms of the normalized cell sizes to range from low in the homogeneous search condition to high in the free viewing condition.

\section{Method}

Apparatus. Two-dimensional orientation of the right eye was measured with an induction coil mounted in a scleral annulus (Skalar Medical, The Netherlands) in an AC magnetic field. This method was first described by Robinson (1963) and refined by Collewijn, Van der Mark, and Jansen (1975). An Apple Macintosh G4/400 equipped with a National Instruments PCI 1200 I/O board presented the stimuli and recorded eye movements and manual responses simultaneously at a sampling rate of $500 \mathrm{~Hz}$. The video card was fast enough to put a stimulus on the screen within one refresh cycle of the monitor. The field generators and lock-in amplifiers were custom built by our technicians and reached the specifications of a Remmel system or better (Remmel, 1984). The maximum error in the signal reached $0.05^{\circ}$. The data were stored on the computer hard disk for offline analysis. The subjects sat between the field coils (Remmel Labs, USA; dimensions $1.0 \times 1.0 \times 1.0 \mathrm{~m}$ ) in front of a Sony E400 19 -in. monitor (distance, $41 \mathrm{~cm}$; resolution, $1,024 \times 768$ pixels; refresh rate, $85 \mathrm{~Hz} ; 256$ gray values). Translations and rotations of 
the head were prevented by a one-axis bite-board. The room was normally lighted during the experiment.

Subjects. Six male (A.K., C.E., E.O., I.H., M.B., and R.W.) and 3 female (E.L., J.Z., and R.O.) observers participated in all three conditions. None showed any visual or oculomotor pathology other than refraction anomalies. The observers had normal or correctedto-normal vision. Subjects C.E., M.B., and R.W. wore corrective glasses. C.E., E.O., and I.H. are the authors of this study; the other subjects were naive as to the goal of the experiment.

Stimuli. Three conditions were presented to the observers, with no practice trials.

Homogeneous search condition: Search task in a homogeneous background. The search area was the uniformly gray monitor screen; this was a uniform stimulus background because, at all scales, each part was the same as any other part. The targets were crosses that consisted of a horizontal and a vertical line of $1 \times 5$ pixels. Some pilot experiments were done to determine suitable gray values for both search area and targets. As a result of the pilot experiments, the luminance of the search area was set at $11.0 \mathrm{~cd} / \mathrm{m}^{2}$ and the luminance of the targets at $9.6 \mathrm{~cd} / \mathrm{m}^{2}$. Each stimulus contained a random number of targets, from one to five, at random positions. There were 48 trials.

Structured search condition: Search task in a picture. A blackand-white group portrait of 15 people, containing parts with much detail as well as parts with little detail, was used as the stimulus background. This was a structured inhomogeneous stimulus background because it contained objects of different scales. Up to nine targets were distributed over the picture. The targets were white crosses on black squares of $9 \times 9$ pixels, and there were 24 trials.

Free viewing condition: Looking at pictures. The stimulus backgrounds for this condition were pictures collected from the Internet. The set of 16 pictures consisted of landscapes, cartoons, photographs, art works, and fractals. Subject E.O. did 23 trials in this condition, and the rest of the observers performed 16.

Procedure. The experiment started with careful placement of the coil on the right eye. The subjects wore the coil for not longer than $30 \mathrm{~min}$, so recording time was limited to about $25 \mathrm{~min}$. Prior to the experiment, a calibration screen was shown to each subject. Five dots had to be fixated one after the other, one in the center of the screen and the others shifted $8^{\circ}$ to the left, right, top, and bottom, respectively. In the homogeneous search and structured search conditions, the subjects were instructed to search for targets until they were confident that they had found them all. The subjects indicated the detection of each target by pressing a button with the right thumb. In the free viewing condition, the task was to look at each stimulus for $30 \mathrm{sec}$. Each trial ended with a push on another button with the left thumb. Preceding each trial, a fixation dot was shown in the center of the screen, and the stimulus appeared when the left button was pushed.

Data analysis. The velocity signal of eye movements was searched for peak velocities above $10 \%$ sec. Each peak was considered a potential indicator of the presence of a saccade. The exact onset of the saccade was determined by going backward in time to the point at which the velocity signal dropped below a level two standard deviations above the average velocity during the stable fixation period before the saccade. The exact offset of the saccade was determined by going forward in time to the point at which the velocity signal dropped below a level two standard deviations above the average velocity during the stable fixation period after the saccade. This method was adopted from an earlier study by Van Der Steen and Bruno (1995). The onsets and offsets of the saccades were used to calculate saccade direction, saccade amplitude, fixation time, and location of fixation. In our further analyses, we rejected fixations shorter than $20 \mathrm{msec}$ and saccades with amplitudes less than $0.1^{\circ}$.

\section{Results}

Mean saccade amplitudes across all subjects were $9.3^{\circ}$, $7.5^{\circ}$, and $6.1^{\circ}$ for the homogeneous search, structured search, and free viewing conditions, respectively. Standard deviations were $7.6^{\circ}, 5.9^{\circ}$, and $5.0^{\circ}$. Mean fixation durations across all subjects were 258,222 , and $270 \mathrm{msec}$ for the homogeneous search, structured search, and free viewing conditions, respectively. Standard deviations were 196, 170, and $225 \mathrm{msec}$. Subjective visual evaluation of the uniformity of fixation density confirmed our expectations for the three conditions. Viewing pictures yields very inhomogeneous fixation densities, with some parts of the stimulus being fixated repeatedly, and others not at all. Search in a homogeneous stimulus, on the other hand, yields fixation locations that are spread about evenly over the total stimulus area. For each individual trial, we computed cell sizes. Representative scan paths for individual trials in each condition are shown in Figure 3, as well as the Voronoi diagrams. Small cells appear in regions of the stimulus where subjects fixated often, and large cells appear in regions where subjects fixated only a few times. The cells in Figure 3A are approximately of the same size, whereas the cells are more differently sized in Figure 3C. Fixations seem to be concentrated in parts of the stimulus that are rich in detail, including at the edges and in color-
A

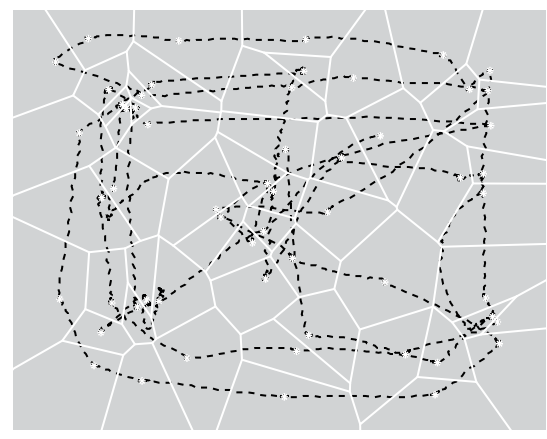

B

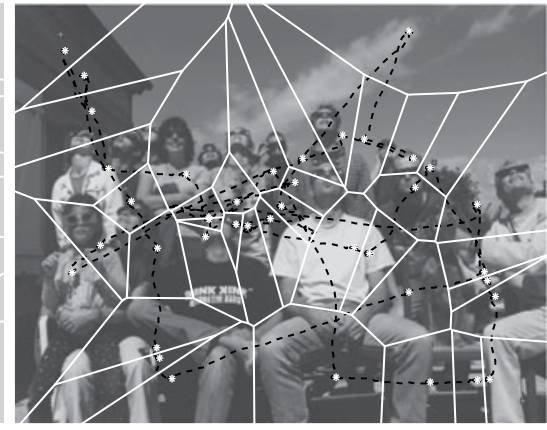

C

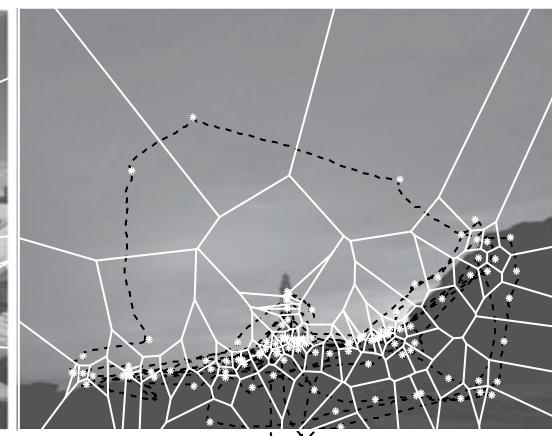

Figure 3. Examples of stimuli, with scan paths (dashed lines), fixation locations (white asterisks), and matching Voronoi diagrams (white lines) superimposed for each of the three conditions. (A) Search in a homogeneous background. (B) Search in a photograph. (C) Free viewing of pictures. 
ful regions. In Figures 3B and 3C, for example, the air is fixated only a few times. In the free viewing condition, the grand average number of fixations per trial was 87 , resulting in a total of about 1,400 fixation locations per subject. In the homogeneous search condition, the grand average number of fixations per trial was 58 , resulting in a total of about 1,400 fixation locations per subject. In the structured search condition, the grand average number of fixations per trial was 32 , resulting in a total of about 1,150 fixation locations per subject.

The skewness measure. The skewness of a data set $x$ can be calculated as follows:

$$
\operatorname{skewness}(x)=\frac{\sum_{i=1}^{N}\left(x_{i}-\mu\right)^{3}}{(N-1) \sigma^{3}},
$$

where $\mu$ is the mean of the data and $\sigma$ is their standard deviation. Figure 4 shows skewness values for the 9 subjects in the three conditions.

Computing the skewness is easy and straightforward, but it has the disadvantage of revealing nothing about the variability of the outcome. A way to obtain a confidence interval is to fit an appropriate probability distribution function to the normalized cell size distribution.

Fitting the cell size distribution. As can be seen from Figure 5, the normalized cell sizes have unimodal distributions of positive noninteger value that are skewed to the right. A number of distributions have these characteristics of unimodality, a positive range of real values, and skewness to the right. Some examples are the chi square, exponential, gamma, log-normal, Rayleigh, and Weibull distributions. We will only consider the gamma, log-normal, and Weibull distributions, since the other three are special cases of either the gamma or the Weibull distribution. The three probability density functions under consideration are

$$
\begin{gathered}
G_{a, b}(x)=\frac{x^{a-1} e^{-x / b}}{\Gamma(a) b^{a}}, \\
W_{\alpha, \beta}(x)=\frac{\beta}{\alpha}\left(\frac{x}{\alpha}\right)^{\beta-1} \cdot \exp \left[-\left(\frac{x}{\alpha}\right)^{\beta}\right],
\end{gathered}
$$

and

$$
L_{\mu, \sigma}(x)=\exp \left[-\frac{1}{2}\left(\frac{\log (x)-\mu}{\sigma}\right)^{2}\right],
$$

where $G_{a, b}$ is the gamma probability density function, $W_{\alpha, \beta}$ the Weibull probability density function, $L_{\mu, \sigma}$ the lognormal probability density function, $a$ the gamma shape parameter, $b$ the gamma scale parameter, $\alpha$ the Weibull scale parameter, $\beta$ the Weibull shape parameter, $\mu$ the mean, $\sigma$ the standard deviation, and $\Gamma$ the gamma function. In Figure 5, the fits of the three different distribution functions are plotted. Subjective visual evaluation of the fits points to the gamma distribution as the most suitable one. An objective measure for goodness of fit is the Kolmogorov-Smirnov test, which tests the probability of data to come from a certain distribution. We applied the Bonferroni correction because we tested the goodness of fit of all three distributions for each data set. The gamma distribution was rejected (total $\alpha=.05$ ) for 11 of the 27 total data sets, whereas the Weibull and log-normal distributions were rejected for 19 of the data sets. Apparently, none of the three proposed distributions fits perfectly to all sets of the data. The literature on Voronoi diagrams indicates that the gamma distribution is an acceptable choice

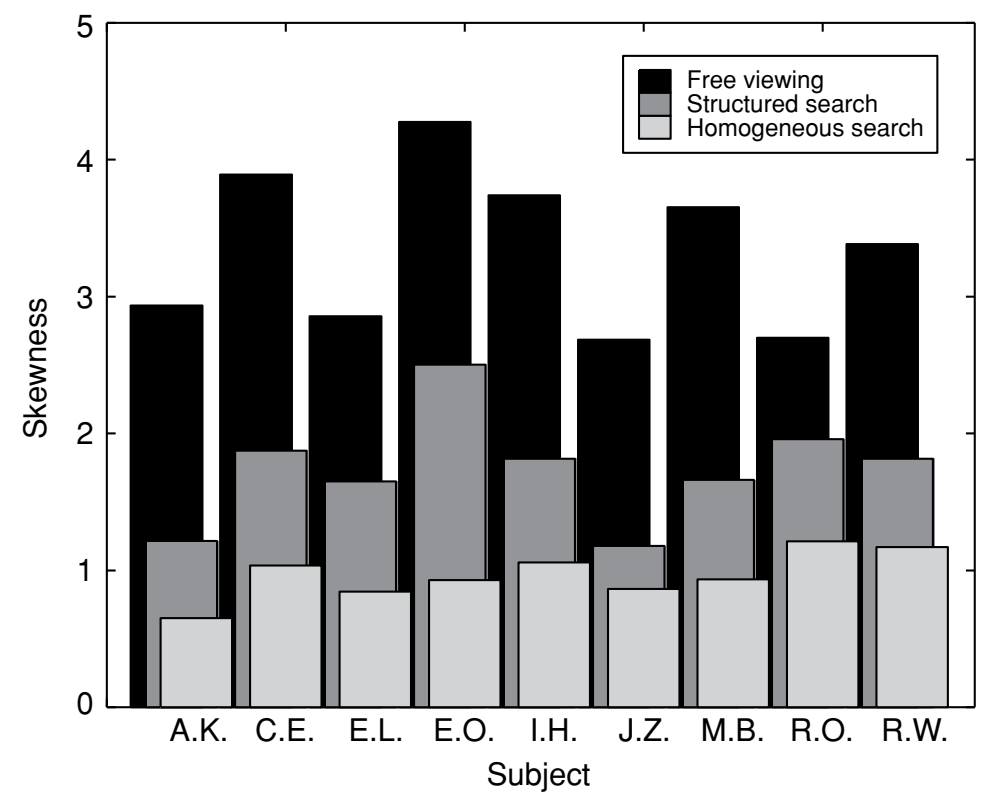

Figure 4. Skewness of normalized Voronoi cell sizes for 9 subjects in the three conditions. 


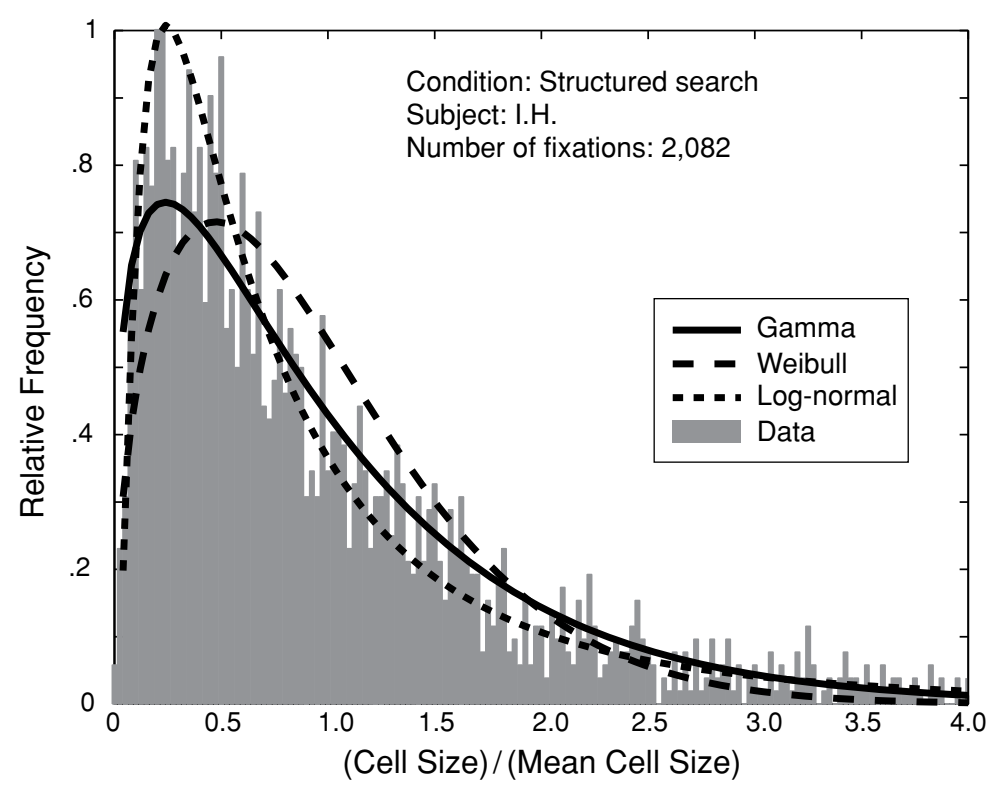

Figure 5. Example of a histogram of the normalized cell sizes and the fits of the three proposed distributions.

for fitting Voronoi cell sizes: It has proven to yield excellent fits of Voronoi cell size distributions calculated for random Poisson processes in extensive simulations (Okabe, Boots, Sugihara, \& Chiu, 1995). Since we did not find a clear preference for any one of the three distributions, we chose the gamma distribution, in accordance with the literature.

The average value of the gamma probability density function is

$$
\mu\left[G_{a, b}(x)\right]=a b,
$$

in terms of its parameters. Because our data were normalized, by definition the average value of the normalized cell sizes is given by

$$
\mu\left(A_{\text {cell }}\right)=1,
$$

where $A_{\text {cell }}$ is the normalized cell size area. The maximumlikelihood fitting procedure (which yields the most likely estimates for the parameters) makes use of the following equality:

$$
\mu\left[G_{a, b}(x)\right]=\mu\left(A_{\text {cell }}\right) .
$$

From Equations 5-7, it then follows that $a=1 / b$. Therefore, normalization has removed one degree of freedom. Only one fitting parameter remains, and Equation 2 can be rewritten as

$$
G_{b}(x)=\frac{x^{1 / b-1} e^{-x / b}}{\Gamma(1 / b) b^{1 / b}} .
$$

Parameter $b$ is positively correlated with the skewness of the distribution: Higher values of $b$ indicate more skewed distributions, corresponding to greater clustering of fixations.

The values for the fitted parameter $b$ according to Equation 8 are plotted in Figure 6. The error bars show the 95\% confidence intervals. The figure shows three properties of $b$ : First of all, the rank order of $b$ for the three conditions is the same for every subject. This is what we expected for the average distribution across subjects. Second, the rank order of $b$ is what we would expect for the three conditions: $b$ is smallest for the homogeneous search condition and largest for the free viewing condition. Third, $b$ shows differences between subjects. This variability may reflect idiosyncratic differences in, for example, search strategy or interests. The fact that $b$ is lowest for Subject E.L. in the homogeneous search condition does not necessarily mean that Subject E.L. should also have the lowest values for $b$ in the other two conditions. The homogeneity of the distribution of fixations does not solely depend on which subject is under investigation; in fact, it is not unlikely that different subjects will react differently under different viewing conditions. In summary, for each subject we found that parameter $b$ was highest in the free viewing condition and lowest in the homogeneous search condition.

\section{Discussion}

In the previous sections, we proposed two measures to describe the uniformity of fixation density: skewness and the gamma fit parameter $b$. Two advantages of the skewness measure are that it is easy to calculate and independent of the distribution's goodness of fit to any particular probability density function. On the other hand, the gamma fitting yields a confidence interval for parameter $b$. If the normalized cell sizes are truly gamma distributed, the measures have the relation

$$
\text { skewness }=2 * \sqrt{b} .
$$

The skewness computed indirectly from $b$, using Equations 8 and 9, differs considerably from the skewness computed directly from the data using Equation 1. This is probably caused by the fact that the normalized cell sizes 


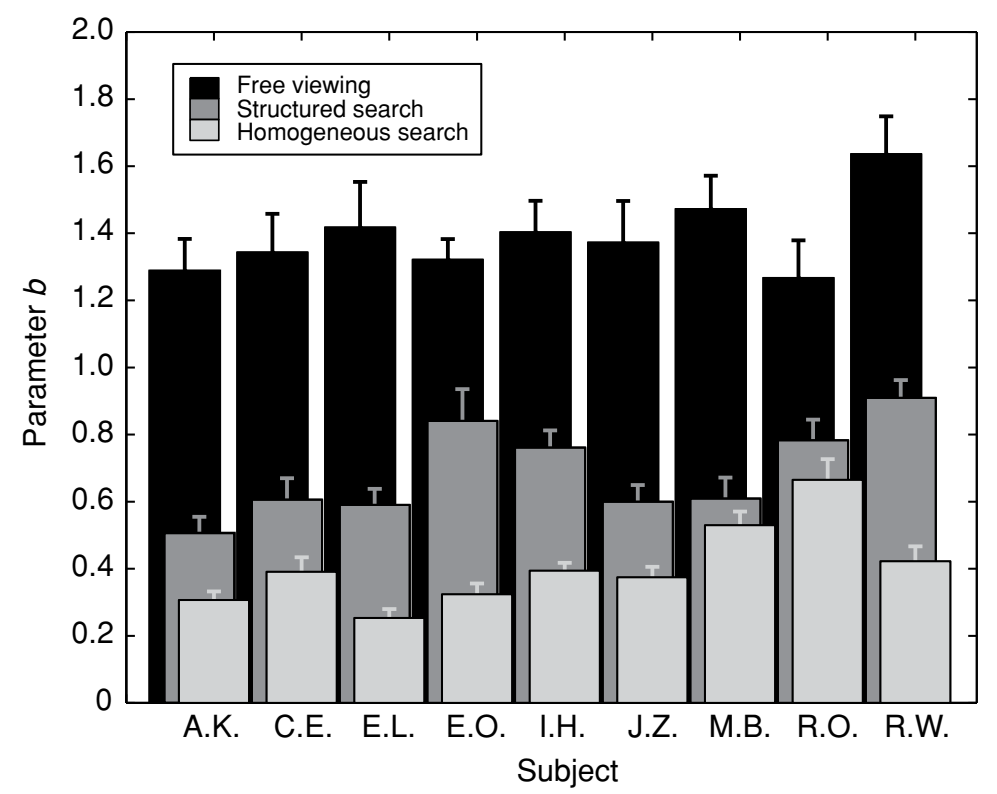

Figure 6. Parameter $b$ for 9 subjects in the three conditions. The error bars show the upper limit of the $95 \%$ confidence intervals.

do not exactly follow a gamma distribution. The difference between the two results may be caused by overestimation of small $b$ values and underestimation of large $b$ values by the fitting algorithm, or by a too-strong influence of extreme values when skewness is calculated with Equation 1.

We found the rank order of the skewness measure and parameter $b$ to be consistent for the three conditions and for all subjects. As we expected, there are also some differences between subjects. The rank order of the skewness measure and $b$ confirms our expectations for the outcome of the Voronoi method for the three fixation densities. Therefore, the results of the Voronoi method are in accord with subjective judgments of the uniformity of fixation density. The Voronoi method, however, has two advantages over a subjective judgment of the clustering of fixations: Its results are objective as well as quantitative, and therefore may be more powerful than intuitive judgments in distinguishing between distributions. The present results give us reason to believe that the Voronoi method is a useful tool for the classification of fixation patterns, such as for discriminating the eye movements of patients from those of healthy people.

\section{SIMULATIONS}

To get a feeling for the possible outcomes of the Voronoi method and the requirements that need to be met in order to apply it to eye movement data, we performed several simulations. Simulations are useful because all conditions are under control, so all properties of a method can be examined systematically. We will only evaluate the results in terms of parameter $b$, since the skewness measure has no confidence interval.
To investigate the range of possible values for $b$, we tried to generate sets of fixation locations that yield extreme values for $b$, as low and as high as possible. The simulation concerned sets of 25 fixation locations. First, in a random set of fixation locations, one fixation was moved so that the value for $b$ was decreased. Changing fixation locations in this way over and over again showed that $b$ can approach 0 , although not reach it. The distribution of fixations resulting from this procedure yielded only Voronoi cells of the same size (Figure 7A). A histogram of the cell sizes would look like a delta peak, and therefore not skewed. Second, a random set of fixation locations was changed in such a way that $b$ increased. In our simulations, there seemed to be no upper limit to the value of $b$. As $b$ increased, the distribution yielded many increasingly small cells, as well as some very large ones (Figure 7E). A histogram of these cell sizes would be a broad, skewed graph. In Voronoi diagrams from real eye movement data, the Voronoi cells cannot be of zero or infinite size. Therefore, the distribution of cell sizes can never be so skewed that an infinite value for $b$ is found. On the other hand, a value of 0 for $b$ is very unlikely, because all cells would have to be exactly the same size. Figure 7 also shows three other values for $b$ and their Voronoi diagrams (panels B-D), corresponding to more common distributions of fixations with intermediate $b$ values. As is clear from Figure 7E, $b=5$ already seems to be an extreme value in terms of real eye movement data. Such a value would mean that the largest part of the stimulus was completely ignored. In conclusion, $b$ values close to 0 correspond to cells of approximately the same size, and therefore fixations that are spread evenly over the total stimulus area. Higher values correspond to a range of different cell sizes: small cell sizes for clustered fixations, and large cell sizes 
A

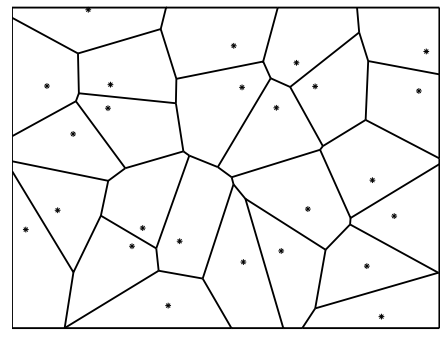

$b=0.01$

D

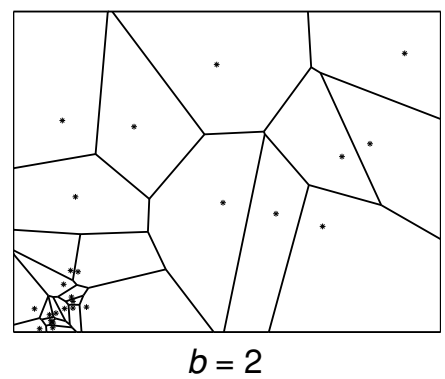

B

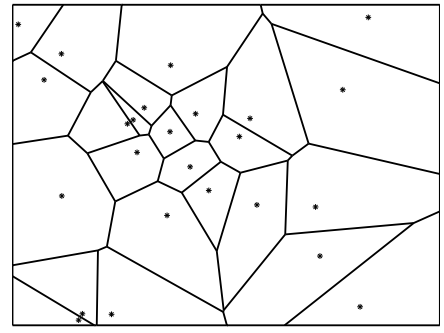

$b=0.5$

E

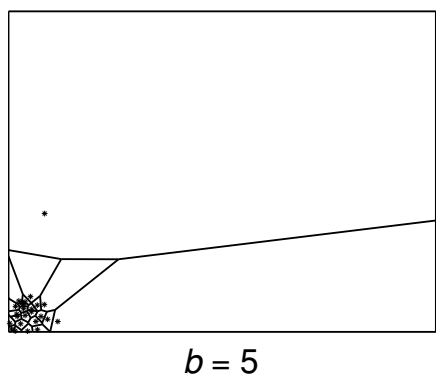

C

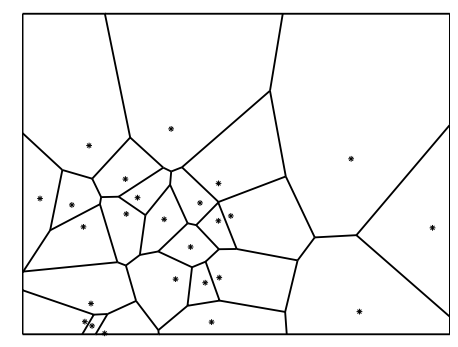

$b=1$

Figure 7. Fixation distributions and their Voronoi diagrams for a range of $b$ values. Higher $b$ values go with more grouped fixations.

for fixations that are remote from the others. The simulations show that $b$ may take any positive real value.

When designing an experiment, it is important to know in advance how many fixations and trials are needed to guarantee a certain confidence interval for parameter $b$. We investigated how the accuracy of the fixation distribution obtained from the Voronoi method depends on the mean number of fixations per trial and the number of trials. We simulated fixation locations and investigated how the $95 \%$ confidence interval for the fitted parameter $b$ changed for different numbers of trials. We also took into account the mean number of fixations per trial and the uniformity of fixation density. For the fixation density we investigated two conditions: (1) uniform fixation distribution and (2) Gaussian fixation probability around the center of the stimulus with variance of about the stimulus dimensions. The simulated stimulus area was $1,024 \times 768$ pixels. Although the resolution is not of great importance for the result of the simulations, the resolution of $1,024 \times 768$ pixels is widely used for stimuli. Locations falling outside the area of $1,024 \times 768$ pixels were rejected and replaced by newly generated locations. Figure 8 shows the number of trials as a function of the mean number of fixations per trial that are needed to obtain $95 \%$ confidence intervals that are $20 \%, 10 \%$, or $5 \%$ of the value of the fitted parameter $b$. For fixation locations from a uniform distribution, the curves in Figure $8 \mathrm{~A}$ can be described by the equation

$$
N_{\mathrm{t}} \times N_{\mathrm{f}} \times 10^{k},
$$

where $N_{\mathrm{t}}$ is the required number of trials, $N_{\mathrm{f}}$ the mean number of fixations per trial, and $k$ a constant. For a confidence interval of $20 \%, k=2.8$; for a confidence interval of $10 \%, k=3.4$; and for a confidence interval of $5 \%, k=$ 4.0. This relationship between the size of the confidence interval and the value of $k$ means that about 1,000 fixations per condition suffice to obtain a confidence interval of $20 \%$, and about 10,000 per condition to obtain a confidence interval of $5 \%$. For fixation locations from a Gaussian distribution about the center of the stimulus, we find that fewer trials are needed to reach the same accuracy if the average number of fixations per trial is more than about 100 .

Referring to Figure 8 shows that the total numbers of fixations in the eye movement experiment were sufficient to achieve $95 \%$ confidence intervals for $b$ smaller than $20 \%$ of its value. For intervals that are $20 \%$ of the value of $b$, a total number of about 1,000 fixations is needed. When standard errors are preferred, note that standard errors are smaller than half of the $95 \%$ confidence intervals.

\section{CONCLUSIONS}

We developed a method to calculate a quantitative measure for the uniformity of fixation density. This method was tested in three conditions that yielded subjectively different spatial distributions of fixations. On the basis of logical reasoning, we predicted the outcome of the Voronoi method. Agreement of our subjective judgments with the outcome of the Voronoi method indicates that our proposed quantitative measure may be a useful tool for objective description of the spatial features of eye movement data.

The result of our Voronoi method is a single parameter, either the skewness measure or $b$. Either parameter 
A

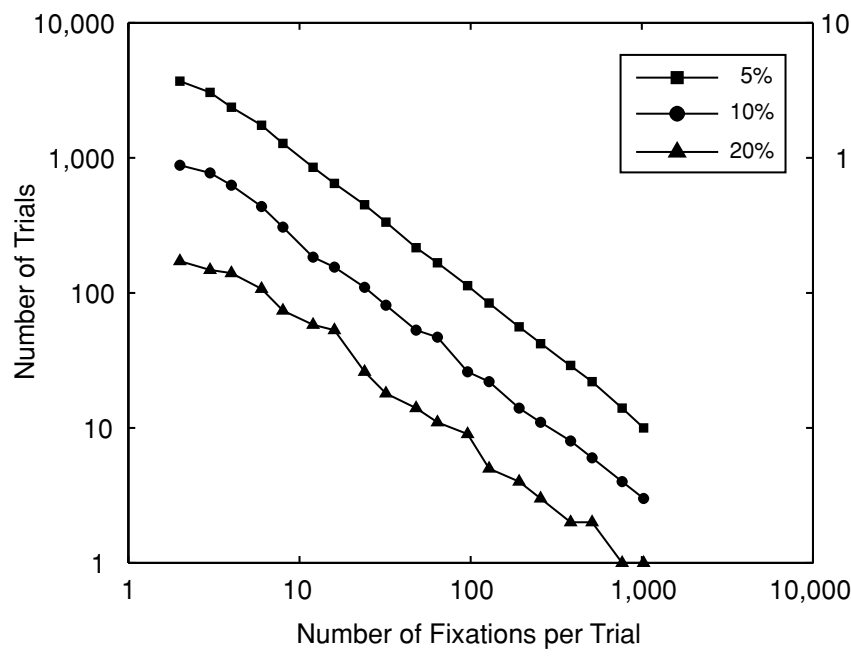

B $\quad$ Variance $<$ Stimulus Dimensions

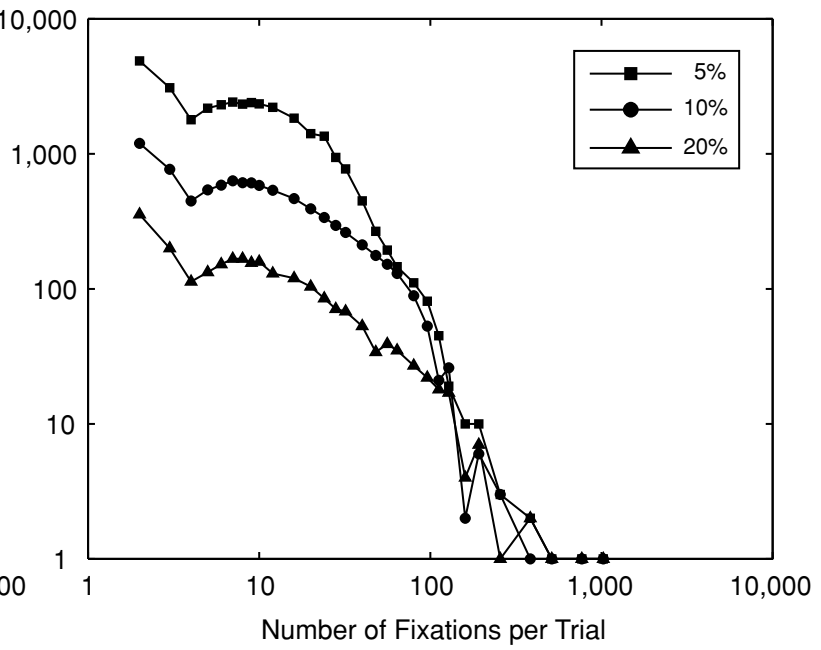

Figure 8. The number of trials as a function of the mean number of fixations per trial needed to obtain $95 \%$ confidence intervals for parameter $b$ that are $20 \%, 10 \%$, or $5 \%$ of its value. (A) With fixations spread randomly over the stimulus (uniform distribution). (B) With fixations located around the center of the stimulus (Gaussian probability with variance that is smaller than stimulus dimensions).

has a simple interpretation: It is positively related to the clustering of fixations. In order to get accurate results, a large total number of fixations must be analyzed. As a rule of thumb, one should use at least 10,000 fixation locations for high accuracy, whereas 1,000 fixation locations suffice for low accuracy. Another major advantage of the Voronoi method is that voluminous amounts of eye movement data can be analyzed relatively easily. Disadvantages are that just a part of the available information in the data is used and that only one parameter remains. Furthermore, the Voronoi method does not address any temporal aspect of viewing behavior.

Although it is not very often used, a simple measure like the nearest-neighbor distance between fixation locations could also provide information about the clustering of fixations and the uniformity of fixation density. The mean nearest-neighbor distance, however, is a measure based on a one-dimensional characteristic, whereas the Voronoi method is based on two-dimensional areas. The Voronoi method has two advantages in comparison with the nearest-neighbor distance. First of all, each fixation location is associated with a unique cell area. In the case of two fixation locations that are very close together, the nearest-neighbor distance will yield the same result for both fixation locations, whereas the Voronoi method will yield different cell areas, since other fixation locations are also taken into account in the computing of the cell areas, not just the nearest fixation location. In short, two-dimensional areas provide more information than do one-dimensional distances. Even in the case of a onedimensional stimulus, such as a line of text, this principle still holds: The nearest-neighbor distance for each fixation location is related to only one other fixation location (the nearest), whereas the Voronoi cell size is based on the distance to two neighboring fixation locations, one on either side. The Voronoi method therefore conforms to the number of dimensions of the stimulus: Cell size is expressed in a one-dimensional distance when the stimulus is one-dimensional and in a volume when the stimulus is three-dimensional. The nearest-neighbor distance is always one-dimensional, regardless of the dimensions of the stimulus. Second, each cell in the Voronoi diagram can be uniquely related to one fixation location, and therefore to its corresponding visual span (Bertera \& Rayner, 2000; Jacobs, 1986; Lévy-Schoen, O’Regan, Jacobs, \& Coeffé, 1984; Pomplun, Reingold, \& Shen, 2001; Reingold, Charness, Pomplun, \& Stampe, 2001). Each cell area may be an indicator for the size of the visual span at that particular location. A part of a stimulus that has a high information density, for instance as a result of many color and contrast differences, is fixated frequently and has many fixations close to each other. In such a part of the stimulus, the Voronoi cell sizes are generally small, which is consistent with the finding that the visual span is smaller as stimulus density increases (Motter \& Belky, 1998).

Voronoi diagrams are part of a large family of tessellations that could also be used to construct areas associated with fixation density. For example, Delaunay triangulation (Delaunay, 1934) produces triangles such that no fixation locations are contained within any triangle's perimeter. However, these triangular areas are not as easily interpretable as the cells in a Voronoi diagram. Areas constructed by Delaunay triangulation belong to three fixation locations, because they are triangles, and therefore contain information about three fixation locations. With Delaunay triangulation, each fixation location might relate to any number of triangles, whereas in Voronoi diagrams, each fixation location relates to exactly one cell. The direct coupling of Voronoi cells with fixation locations also provides an objective way to relate fixation locations to local image content. Each fixation location belongs to a certain part of the stimulus - namely, the part that is con- 
tained by its Voronoi cell. Image analysis might be used on each Voronoi cell to try to establish correlations between any frequently used parameter in image analysis, such as contrast or brightness, and characteristics such as fixation duration or the size of the Voronoi cell.

Recent research (Vlaskamp, Hooge, \& Over, 2003) supports the idea that the Voronoi method is a powerful tool to analyze uniformities of fixation densities. In that study, 6 subjects participated in a search experiment under three stimulus conditions in which the contrast of objects with the background was either consistently high, consistently low, or a mixture of high, medium, and low. In the high- and low-contrast conditions, the fixation density across the stimulus was highly uniform, resulting in (low) values for $b$ ranging from 0.15 to 0.22 . In the mixedcontrast condition, the fixation density was less uniform across the stimulus, resulting in values for $b$ ranging from 0.20 to 0.29 . For each subject, $b$ was higher in the mixedcontrast condition than in the other two conditions. The uniformity of fixation density was practically the same in the high- and low-contrast conditions, although the number of fixations per trial differed considerably. Thus, even with target-absent stimuli, in which subjects had to search the entire stimulus to realize that there was no target, the Voronoi method yielded results that were congruent with the visual evaluations and was able to distinguish between different uniformities of fixation density: The densities were less uniform in the mixed-contrast condition than in either the high- or the low-contrast condition. Visual evaluation required a substantial amount of time and was subjective, whereas the Voronoi method could relatively easily quantify uniformities.

\section{REFERENCES}

Aks, D. J., Zelinsky, G. J., \& Sprott, J. C. (2002). Memory across eye-movements: $1 / f$ dynamic in visual search. Nonlinear Dynamics, Psychology, \& Life Sciences, 6, 1-25.

Bertera, J. H., \& RAYNer, K. (2000). Eye movements and the span of the effective stimulus in visual search. Perception \& Psychophysics, 62, 576-585.

BRANDT, S. A., \& Stark, L. W. (1997). Spontaneous eye movements during visual imagery reflect the content of the visual scene. Journal of Cognitive Neuroscience, 9, 27-38.

Choi, Y. S., Mosley, A. D., \& Stark, L. W. (1995). String editing analysis of human visual search. Optometry \& Vision Science, 72, 439-451.

Collewijn, H., Van der Mark, F., \& Jansen, T. C. (1975). Precise recording of human eye movements. Vision Research, 15, 447-450.

De Gennaro, L., Ferrara, M., Curcio, G., \& Bertini, M. (2001). Visual search performance across $40 \mathrm{~h}$ of continuous wakefulness: Measures of speed and accuracy and relation with oculomotor performance. Physiology \& Behavior, 74, 197-204.

Delaunay, B. (1934). Sur la sphere vide. Bulletin of Academy of Science USSR, 7, 793-800.

De Lucio, J. V., Mohamadian, M., Ruiz, J. P., Banayas, J., \& BerNALDEZ, F. G. (1996). Visual landscape exploration as revealed by eye movement tracking. Landscape \& Urban Planning, 34, 135-142.

Gbadamosi, J., \& Zangemeister, W. H. (2001). Visual imagery in hemianopic patients. Journal of Cognitive Neuroscience, 13, 855-866.

Hooge, I. T. C., \& Erkelens, C. J. (1999). Peripheral vision and oculomotor control during visual search. Vision Research, 39, 1567-1575.
JACOBS, A. M. (1986). Eye-movement control in visual search: How direct is visual span control? Perception \& Psychophysics, 39, 47-58.

LAENG, B., \& TeODORESCU, D.-S. (2002). Eye scanpaths during visual imagery reenact those of perception of the same visual scene. Cognitive Science, 26, 207-231.

Levi, D. M., Klein, S. A., \& Aitsebaomo, A. P. (1985). Vernier acuity, crowding and cortical magnification. Vision Research, 25, 963-977.

Lévy-Schoen, A., O'Regan, J. K., Jacobs, A. M., \& Coeffé, C. (1984). The relation between visibility span and eye movements in various scanning tasks. In A. G. Gale \& F. Johnson (Eds.), Theoretical and applied aspects of eye movement research: Selected/edited proceedings of the Second European Conference on Eye Movements, Nottingham, England, 19-23 September, 1983 (pp. 133-142). Amsterdam: North-Holland.

Motter, B. C., \& BelKy, E. J. (1998). The zone of focal attention during active visual search. Vision Research, 38, 1007-1022.

Noton, D., \& Stark, L. (1971a). Scanpaths in eye movements during pattern perception. Science, 171, 308-311.

Noton, D., \& StaRK, L. (1971b). Scanpaths in saccadic eye movements while viewing and recognizing patterns. Vision Research, 11, 929-942.

Okabe, A., Boots, B., Sugihara, K., \& Chiu, S. N. (1995). Spatial tessellations: Concepts and applications of Voronoi diagrams. New York: Wiley.

Pelz, J., Hayhoe, M., \& Loeber, R. (2001). The coordination of eye, head, and hand movements in a natural task. Experimental Brain Research, 139, 266-277.

Phillirs, M. L., \& DaVID, A. S. (1997). Visual scan paths are abnormal in deluded schizophrenics. Neuropsychologia, 35, 99-105.

Pomplun, M., Reingold, E. M., \& Shen, J. (2001). Investigating the visual span in comparative search: The effects of task difficulty and divided attention. Cognition, 81, B57-B67.

Reingold, E. M., Charness, N., Pomplun, M., \& Stampe, D. M. (2001). Visual span in expert chess players: Evidence from eye movements. Psychological Science, 12, 48-55.

Remmel, R. S. (1984). An inexpensive eye movement monitor using the scleral search coil technique. IEEE Transactions on Biomedical Engineering, 31, 388-390.

RoBinson, D. A. (1963). A method of measuring eye movements using a scleral search coil in a magnetic field. IEEE Transactions on Biomedical Engineering, 10, 137-145.

Shinoda, H., Hayhoe, M. M., \& Shrivastava, A. (2001). What controls attention in natural environments? Vision Research, 41, 35353545.

Van Der Steen, J., \& Bruno, P. (1995). Unequal amplitude saccades produced by aniseikonic patterns: Effects of viewing distance. Vision Research, 35, 3459-3471.

Vlaskamp, B. N. S., Hooge, I. T. C., \& Over, E. A. B. (2003). Local contrast affects fixation density in saccadic search [Abstract]. Perception, 32(Suppl.), 109B.

VoRONOI, G. (1908). Nouvelles applications des paramètres continus à la théorie des formes quadratiques: Deuxième mémoire. Recherches sur les paralléloèdres primitifs. Journal für die Reine \& Angewandte Mathematik, 134, 198-287.

Westheimer, G., \& Hauske, G. (1975). Temporal and spatial interference with Vernier acuity. Vision Research, 15, 1137-1141.

WiLliams, L. G. (1966). The effect of target specification on objects fixated during visual search. Perception \& Psychophysics, 1, 315-318.

YARBUS, A. (1967). Eye movements and vision. New York: Plenum.

Zangemeister, W. H., Sherman, K., \& Stark, L. (1995). Evidence for a global scanpath strategy in viewing abstract compared with realistic images. Neuropsychologia, 33, 1009-1025.

Zelinsky, G. J., Rao, R. P. N., Hayhoe, M. M., \& Ballard, D. H. (1997). Eye movements reveal the spatiotemporal dynamics of visual search. Psychological Science, 8, 448-453.

Zihl, J., \& Hebel, N. (1997). Patterns of oculomotor scanning in patients with unilateral posterior parietal or frontal lobe damage. Neuropsychologia, 35, 893-906.

(Manuscript received October 11, 2004; revision accepted for publication February 15, 2005.) 